\title{
The Partitioning of Octyl Phenol Ethoxylate Surfactant between Water and Sunflower Oil
}

\author{
Jozua Laven*, Daniela Senatore, Wim K. Wijting and Gijsbertus de With
}

Laboratory of Materials and Interface Chemistry, Eindhoven University of Technology, The Netherlands

\begin{abstract}
The partitioning of a micelle-forming octyl phenol ethoxylate surfactant (Triton X-100) between water and sunflower oil was investigated and quantified as partition coefficient $P$, i.e. the ratio of total surfactant concentrations (molecules dissolved and in micelles) in oil and water. Values of $P$ were determined over 5 decades of the concentration in the aqueous phase. Below the critical micelle concentration $(\mathrm{cmc}), P=2.0$ was found to be independent of concentration over a range of more than two decades. When adding surfactant to an oil-water two-phase system at or beyond the $\mathrm{cmc}$, all extra surfactant accumulates in the water phase, and the value of $P$ reduces accordingly. Arguments are given why emulsification leads to an $\mathrm{O} / \mathrm{W}$ emulsion while Bancroft's rule as based on the molecular preference of the surfactant for oil $(P=2.0)$, would suggest the opposite. Bancroft's rule should be taken in its original form, taken into account all oil in both phases.
\end{abstract}

Keywords: Partition coefficient, Emulsification, Triton X-100, Critical micelle concentration, HLB.

\section{INTRODUCTION}

The classical rule of Bancroft [1] is still a useful first order guide for predicting what will become the continuous phase (e.g., oil, in a W/O emulsion) when mixing oil and water in the presence of surfactant: it is the phase in which the surfactant is best soluble. In the hydrophilic-lipophilic balance (HLB) concept and theory this was understood by the notion that, due to Marangoni flows, touching droplets in such an emulsion are more stable against film rupture than droplets in the reverse type of emulsion [2-6]. Note that for micro emulsions, an important additional factor is how the surfactant in equilibrium is organized at the interface, leading to a preferred curvature $[1,7,8]$.

For regular emulsions, the final emulsion type will also be affected by factors like the volume fractions of both phases, as schematically depicted in Fig. (1). In the ambivalent area in this Figure the final emulsion type is especially determined by the processing step. In this area phase inversion (from $\mathrm{O} / \mathrm{W}$ to $\mathrm{W} / \mathrm{O}$ and vice versa) can occur [9], either transitionally $[10,11]$ or catastrophically [10, 12-14].

As pointed out by Ruckenstein [15], the Bancroft rule only applies at moderate surfactant concentrations. At too high levels a black film may be generated and the rupture of such a film, via a nucleation step, may be the ratedetermining step of the coalescence. At low surfactant concentrations the surface concentration gradient cannot generate sufficiently strong Marangoni flows and the emulsion type is controlled by the mixing (hydrodynamic process). Ruckenstein argued that the violations to the rule of Bancroft

*Address correspondence to this author at the Eindhoven University of Technology, Building HEO 2.42, PO box 513, 5600 MB Eindhoven, The Netherlands; Tel: +31-402473682; Fax: +31-402445619;

E-mail: J.Laven@TUE.NL as found by Binks [16] are not violations because the surfactant concentration was low.

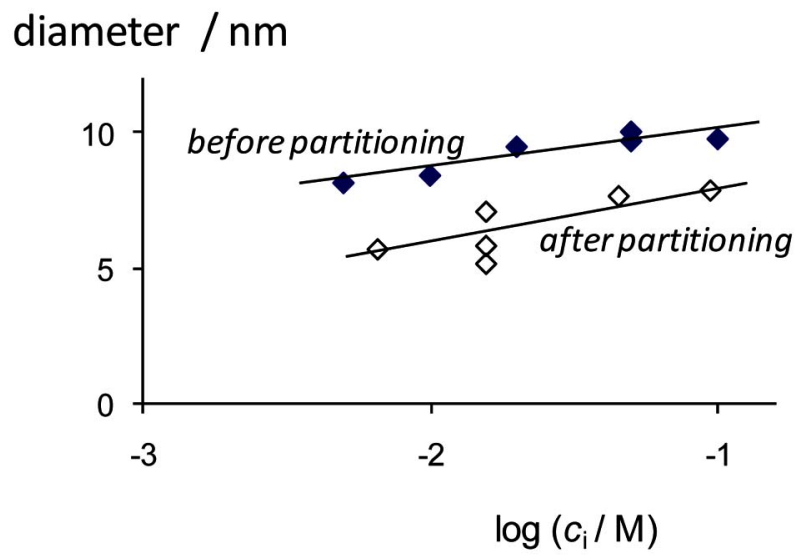

Fig. (1). The effects of volume fraction of oil $\varphi_{0}$ and the HLB value on the final emulsion, schematically.

For many oil/water systems, partition coefficients of numerous surfactants have been investigated, especially for water/alkane systems [17]. A particular surfactant, octyl phenol ethoxylate emulsifier (Triton X-100), is used in a very wide range of cleaning compounds and also for more sophisticated applications in biochemistry research like in purification of high-copy plasmid DNA, in several protein separation protocols and in Antibody Blocking Buffer for immune-fluorescence [18]. Whereas Triton X-100 partitioning between water and a few alkanes has been investigated $[19,20]$, albeit below the critical micelle concentration $(\mathrm{cmc})$, no such data are available for triglycerides as oil phase (the common fat of animals and plants), neither below nor beyond the $\mathrm{cmc}$. The present paper reports on the partitioning of this specific surfactant between sunflower oil and water, 
both below and above the cmc. The relation for this surfactant between its partition coefficient and micellar behavior with its emulsifying function is discussed.

\section{EXPERIMENTAL}

Solutions of Triton X-100 UltraSigma (hydrophobic group: 4-(1,1,3,3-tetramethylbutyl)-phenyl; hydrophilic group: $\left(\mathrm{C}_{2} \mathrm{H}_{4} \mathrm{O}\right)_{\mathrm{n}}$ with $\mathrm{n}=9.5$; obtained from Sigma-Aldrich; $\mathrm{HLB}=13.5[21] ; M=625 \mathrm{Da}$; mass density $1070 \mathrm{~kg} / \mathrm{m}^{3}$ ) over a wide range of molarities were made in deionized water (Millipore Milli-Q). Glass bottles of $250 \mathrm{~mL}$ bottles were filled with $50 \mathrm{~g}$ of any of these solutions together with $50 \mathrm{~g}$ of sunflower oil (Fluka; as received; mass density 922 $\mathrm{kg} / \mathrm{m}^{3}$ ). The closed bottles were rolled on a bottle roller at approximately $15 \mathrm{rpm}$ for $12 \mathrm{~h}$ at $20{ }^{\circ} \mathrm{C}$. After a rest period of typically 5 days, the Triton partitioning between water and oil in each bottle was determined by comparing the Triton X100 concentrations in the aqueous phase before and after partitioning, using UV spectroscopy. UV spectra were collected with a HP-8453 UV-VIS Spectrometer, using $1 \mathrm{~cm}$ optical path cuvettes. Measuring the surfactant concentration in the oil phase was not well possible due to the high UV absorption by the oil itself. The amount of Triton X-100 that had disappeared from the water phase was supposed to reside in the oil phase. In the calculations involved it was assumed that after surfactant partitioning, all oil and water molecules reside in the oil and water phases, respectively, and that the volume of any surfactant-solvent mixture is equal to the summed volume of the pure components.

Surface tensions were determined at $20{ }^{\circ} \mathrm{C}$ with a Krüss Tensiometer K10T equipped with a $2 \mathrm{~cm}$ wide platinum Wilhelmy plate, according to ISO 304 [22]. Light scattering experiments for checking the presence of micelles were conducted with $10 \times 10 \mathrm{~mm}$ cuvettes in a Malvern Zetasizer Nano-ZS. This equipment deviated from the standard configuration in that it is equipped with a $632 \mathrm{~nm}$ monochromatic filter just in front of the detector, in order to suppress any signal coming from fluorescence. Diffusion coefficients were derived with the Contin procedure [23] contained in the software of the equipment while particle sizes were derived thereof as based on the Stokes-Einstein equation for spheres using the viscosity of pure water.

\section{RESULTS AND DISCUSSION}

For each aqueous Triton X-100 solution prepared for partitioning experiments, the initial surfactant concentration was determined by using a calibration curve that was obtained from spectra of a series of aqueous Triton X-100 solutions, as shown in Fig. (2). It can be seen that the absorption $A$ is linear with the concentration $c$, since $A / c$ in Fig. (2), is virtually constant $\left(A=c^{1.0026}\right)$ for the area of the composed peak between 260 and $290 \mathrm{~nm}$ after correction by a straight base line between the limits. It was checked that the partitioning results to be presented are not sensitive to where the peak limits are exactly positioned on varying each limit position by $+/-10 \mathrm{~nm}$. The peak around $225 \mathrm{~nm}$ might at first sight appear more useful because of the larger signal. However, a transition in absorption behavior, still not understood, can be seen between the sub-micellar range and the micellar range (at $\sim 1 \mathrm{mM}$ ). This makes that absorption band unsuitable for our purposes.

The partitioning of Triton X-100 between contacting oil and water domains is shown in Fig. (3), as the ratio $c_{\mathrm{O}} / c_{\mathrm{W}}$ of its concentrations in the oil and aqueous phases after partitioning. It can clearly be seen that below the $\mathrm{cmc}$ the partition coefficient $c_{\mathrm{O}} / c_{\mathrm{W}}$ is constant at a value $c_{\mathrm{O}} / c_{\mathrm{W}}=2.0$.

This is not unexpected as at such low concentrations solutions easily behave ideally, both in oil and in water, thereby obeying Henry's law [24]. The consistency of our $c_{\mathrm{O}} / c_{\mathrm{W}}$ data below the cmc is much better than that of the data for water/alkane systems published in the literature $[19,20]$.

In Fig. (3), are also shown surface tensions of regular Triton X-100 solutions in water and of the water phase after partitioning, both plotted as a function of the actual aqueous Triton X-100 concentration. The difference between both surface tension series is not fully clear but may be related to a number of phenomena. First, a commercial sample is not perfectly monodisperse in molar mass and molecular composition; some fractionation may have occurred while partitioning, making the average character of the Triton X-100 left in the bulk water phase less hydrophobic. Additionally, traces of oil and fatty acids thereof will dissolve in the water phase

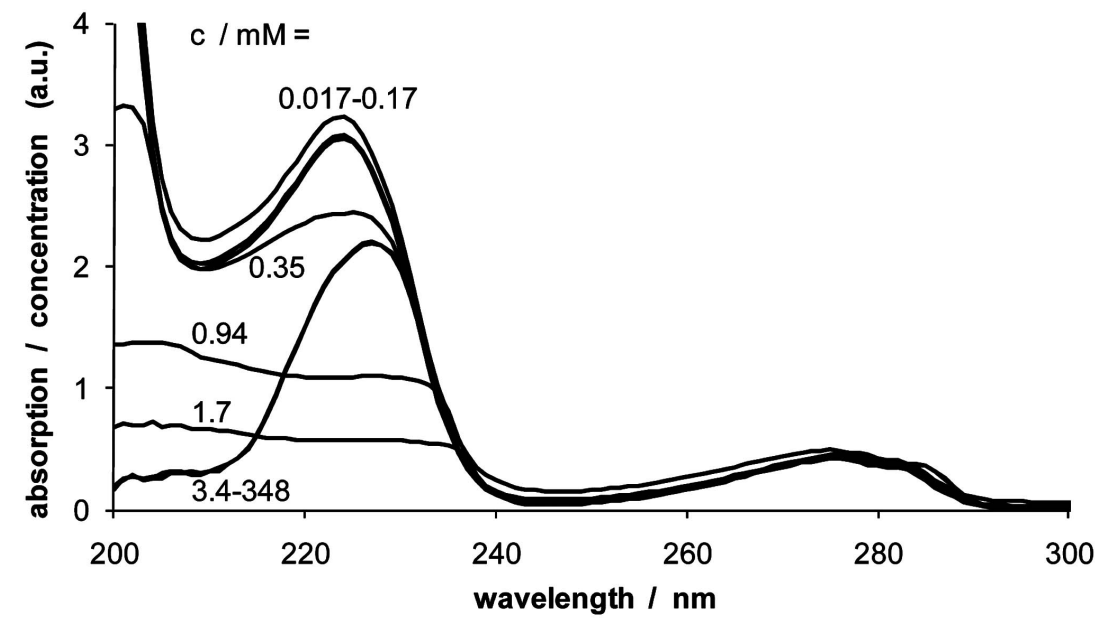

Fig. (2). UV absorption spectra of a series of aqueous Triton X-100 solutions of which the surfactant concentrations in mM are depicted adjacent to the individual curves. The plotted absorption values had been divided by the surfactant concentrations. 


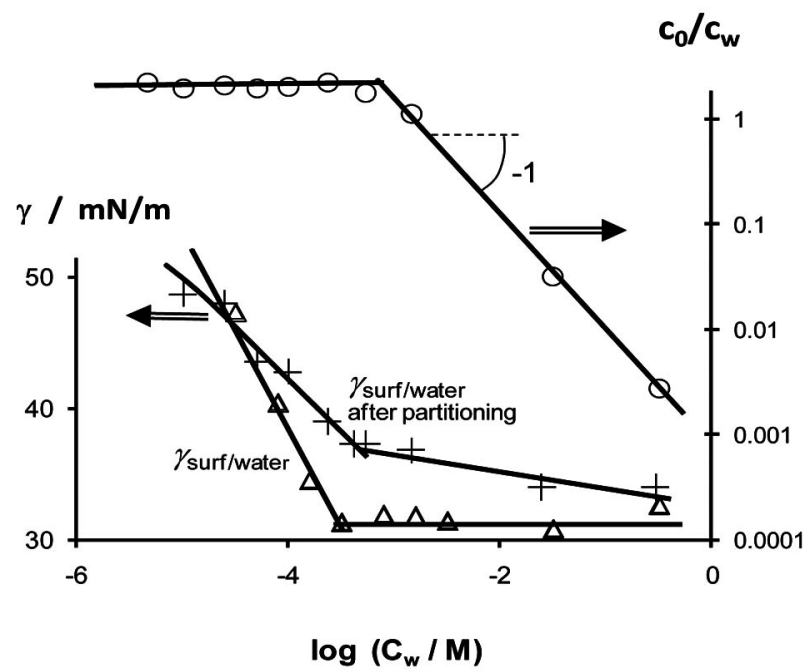

Fig. (3). The equilibrium concentration ratio $c_{\mathrm{O}} / \mathrm{c}_{\mathrm{W}}(\circ)$ of Triton $\mathrm{X}$ 100 in contacting oil and water domains as function of the Triton $\mathrm{X}-100$ equilibrium concentration in the water domain. Also surface tensions of the water phase after equilibration $(+)$ and of regular aqueous Triton $\mathrm{X}-100$ solutions $(\Delta)$ are plotted.

and may have interaction with the dissolved Triton X-100 molecules.

Beyond the $\mathrm{cmc}$ the partitioning is completely different from that below the cmc. The surfactant has an increasing preference for the water phase with increasing concentration. This can be understood as follows. Surfactant that is added to an aqueous solution of that surfactant at or beyond the cmc will in principle accumulate in micelles while leaving the concentration of molecularly dissolved surfactant in water, and thus its activity, unchanged. With a fixed chemical activity one would not expect that any part of the added surfactant will migrate as individual molecules to a contacting oil phase. In agreement with theoretical expectation, the slope $d \ln \left(c_{o} / c_{w}\right) / d \ln c_{w}$ of the partition coefficient curve in Fig. (3), is -1 .

For the two highest points (far beyond $\mathrm{cmc}$ ) the partition coefficients were determined in a slightly different way because the results of the method described earlier appeared inconsistent in this case. The reason is probably that after partitioning virtually all the surfactant was left in the water phase and, when subtracting this amount from the initial amount of Triton X-100, very small relative errors in amounts for the water phase resulted in very large relative errors when calculating concentrations in the oil phase. Also, the measurement of the UV absorption in such an aqueous system was possibly distorted by traces of UV-absorbing oil in the micelles. In these two cases the oil phase after partitioning (O1) was separated from the aqueous phase after partitioning (W1) and the Triton X-100 in O1 was partitioned again in contact with fresh water, giving concentrations in new oil and water phases (O2 and W2). From the Triton concentration in W2, which was below the cmc, the concentration in $\mathrm{O} 2$ could be deduced on the basis of accurate data for the partition coefficient as already found below the cmc. In this way, the concentration in $\mathrm{O} 1$ could be evalu- ated. Knowing the total amount of Triton X-100 used in the first partitioning, its concentration in W1 could now be assessed which permitted the evaluation of the partition coefficient at concentrations beyond the $\mathrm{cmc}$.

In Fig. (4), the micellar diameter in the water phase is plotted both for the initial aqueous surfactant solution and for the aqueous phase after partitioning against sunflower oil. Diameters are plotted as a function of the concentration $c_{\mathrm{i}}$ in the initial surfactant solution. Diameters are derived from diffusion coefficients $D$ according to the StokesEinstein equation using the viscosity of pure water, both before and after partitioning. Obviously using this equation (which requires the diffusivity of a hard sphere at infinite dilution $D_{0}$ and the continuous phase viscosity) is an approximation as e.g. hydrodynamic and long range interactions between droplets at non-zero concentrations affect the diffusion coefficient [25]. Having no reason why these effects would act differently for the two sets of data presented, we conclude that there is no indication that the aqueous micelles are swollen by any appreciable amount of oil.

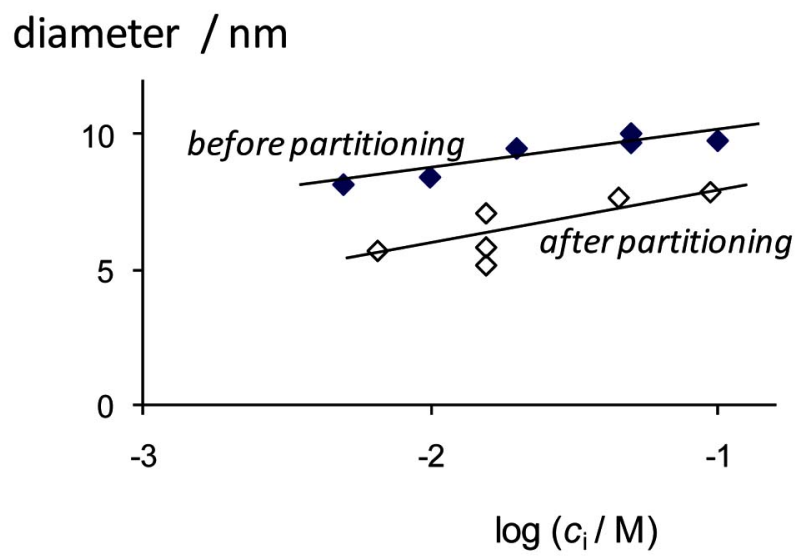

Fig. (4). Micellar diameters in the initial aqueous surfactant solution and in the aqueous phase after partitioning against sunflower oil, as function of the concentration $c_{\mathrm{i}}$ in the initial aqueous surfactant solution. Diameters are derived from diffusion coefficients according to the Stokes-Einstein equation using the viscosity of pure water, both before and after partitioning.

When comparing our results on $P=c_{o} / c_{w}$ to previous results with alkane as oil phase we see that Campanelli et al. [20] found $P \approx 0.14$ with hexadecane and $P=0.08$ with a light mineral oil, in both cases over a concentration range of a factor 5 just below the cmc. Below that range they appeared not to be able to arrive at reliable results. Göbel et al. [19] found, just below the cmc, $P=0.24$ with hexadecane, $P=0.71$ with n-heptane and $P=1.30$ with cyclohexane. Our results with sunflower oil below the cme show a constant value $P=2.0$ over a concentration range of more than two decades below the cmc. The reason for our relatively high value of $P$ is probably that vegetable oil is more polar than the alkanes discussed.

According to Bancroft's rule [1] the present surfactant, its molecules being more soluble in oil than in water, would not be a good emulsifier of sunflower oil in water. However, our experience is that making such $\mathrm{O} / \mathrm{W}$ emulsions is not 
difficult at all. The logic behind Bancroft's rule is that the type of emulsion $(\mathrm{O} / \mathrm{W}$ or $\mathrm{W} / \mathrm{O})$ with the most effective stabilizing Marangoni flow [26] when two droplets approach each other is the most stable one. Such flow is suppressed when sufficient diffusion of surfactant to the interface can take place from the "bulk" of a droplet, the supply rate of the surfactant from within the droplet being approximately proportional both to its diffusivity and its concentration gradient in the droplet near the droplet surface. Such concentration gradient can in a zeroth order approximation be estimated, inspired by boundary layer theory, as the ratio of the concentration in the bulk of the droplet $\left(c_{\mathrm{bd}}\right)$ and the thickness $t$ of the subsurface layer in the droplet that becomes depleted of surfactant that leaves the droplet (we want to focus on the most important aspects and therefore leave out obvious improvements in the model like using the difference in bulk concentrations of the disperse and the continuous phase $\left(c_{\mathrm{bd}^{-}}\right.$ $c_{\mathrm{bc}}$ ) instead of $\left.c_{\mathrm{bd}}\right)$. As emulsification is usually performed well beyond the cmc, a couple of aspects make Bancroft's rule less applicable in such a case.

$i$ The concentration gradient in the subsurfaces of approaching water droplets is larger than expected on the basis of the $\mathrm{cmc}(\sim \mathrm{cmc} / t)$ due to the presence of micelles which can make the boundary layer for molecular diffusion much thinner. The value $c_{\mathrm{dba}} / t$ probably is a much better estimation ( $c_{\mathrm{dba}}$ is the droplet's bulk analytical surfactant concentration), be it possibly too large in view of the finite disassembling time of micelles.

ii As based on the ratio of viscosities of sunflower oil and water, the diffusivity in water is larger by a factor 100 . The combined effect of (i) and (ii) probably leads to the result that $\mathrm{O} / \mathrm{W}$ is still the favored type of emulsion.

Ruckenstein defined two parameters, $R_{1}$ and $R_{2}$, in order to characterize the macro- and micro-emulsification of a surfactant / solvent A /solvent B system ( $\mathrm{S} / \mathrm{A} / \mathrm{B}$ system). $R_{1}$ is the ratio of the affinities of the surfactant to both phases and thus is closely related to its partition coefficient (qualitatively, $R_{1}>1$ corresponds to $P>1$ ). The parameter $R_{2}$ is related to the morphology of the surfactant-packed interface between solvents $\mathrm{A}$ and $\mathrm{B}$ and reflects the preferential curvature of the interface (qualitatively, $R_{2}>1$ corresponds to a preference for the $\mathrm{O} / \mathrm{W}$ type). With micro-emulsions, $R_{1}$ along with $R_{2}$ determine the emulsion type. Ruckenstein quotes Hildebrand and others to indicate that for macroemulsions $R_{1}$ does and $R_{2}$ does not play a role in determining the type. In our case, with no signs of micro-emulsion formation, $R_{1}>1$ which violates practical experience that $\mathrm{O} / \mathrm{W}$ emulsions are formed.

We propose to expand Ruckenstein's rule for microemulsions also to micelle-containing macro-emulsions, in other words to consider micelles in a S/A/B system as hardly or not swollen micro-emulsion droplets. In fact, implicitly Ruckenstein allows this when, at the last page of his paper, he discusses the mechanism of coalescence of macro droplets in a mixed micro/macro-emulsion. Apart from some moderating amendments made by Ruckenstein (not very high or low concentration, surface viscosity, surface diffusion, instability of thin film between droplets by mechanical and thermal perturbations) and remarks like ours on the relative values of viscosities of and diffusivities in both bulk phases, there appears no reason to disqualify Bancroft's original rule. Note that when Bancroft, by 1913, spoke about surface active "hydrophile" and "hydrophobe colloids" [1] he apparently was not aware of any aggregation of them in micelles (by that time the unexpectedly high electric conductivity of metal soaps in water made McBain [27] to suggest the existence of "highly charged colloidal aggregates, micelles or colloidal ions").

Thus, when selecting surfactants for macro emulsions it is in practice more useful to consider the effective partition coefficient $P$ (ratio of analytical concentrations of surfactant molecules, molecularly dissolved as well as in micelles), than to compare the affinities of the surfactant to the water and oil phases, as reflected in the partition coefficient below the cmc.

\section{CONCLUSIONS}

In this paper the partitioning of Triton X-100 between water and sunflower oil was measured over two decades below and three decades beyond the cmc, by making use of the UV absorption by Triton X-100. Below the cmc the partition coefficient (ratio of concentrations in oil and water) is 2.0 , independent of concentration. When going beyond the cmc the concentration in the oil phase remains virtually constant at a level compatible with the cmc in water; any additional surfactant is stored in aqueous micelles. The results are discussed in relation to the oil in water emulsification process and Bancroft's rule. This rule should be taken in the way it was meant originally (compare total amounts of surfactants in both phases) predicting the type of emulsion involved.

\section{ACKNOWLEDGEMENTS}

The authors wish to thank the Foundations Priority Program Materials (PPM), Stichting Chemische Wetenschappen (STW) and Dutch Polymer Institute (DPI) for financial support and Dr. A.N. Zdravkov for stimulating discussions.

\section{REFERENCE}

[1] Bancroft, W.D. The Theory of Emulsification. J. Phys. Chem., 1913, 17, 501-519.

[2] Chesters, A.K. The modeling of coalescence processes in fluidliquid dispersions: a review of current understanding. Chem. Eng. Res. Des., 1991, 69, 259-270.

[3] Mousa, H.; Agterof, W.; Mellema, J. Determination of the orthokinetic coalescence efficiency of droplets in simple shear flow using mobile, partially mobile and immobile drainage models and trajectory analysis. Chem. Eng. Res. Des., 2002, 80, 345-354.

[4] Saboni, S.A.; Gourdon, C.; Chesters, A.K. The influence of interphase mass transfer on the drainage of partially-mobile liquid films between drops undergoing a constant interaction force. Chem. Eng. Sci., 1999, 54, 461-473.

[5] Peters, G.W.M.; Zdravkov, A.N.; Meijer, H.E.H. Transient interfacial tension and dilatational rheology of diffuse polymer-polymer interfaces. J. Chem. Phys., 2005, 122, 104901

[6] Ban, T.; Kawaizumi, F.; Nii, S.; Takahashi, K. Study of drop coalescence behavior for liquid-liquid extraction operation, Chem. Eng. Sci., 2000, 55, 5385-5391.

[7] Griffin, W.C. Classification of Surface-Active Agents by "HLB“.. J. Soc. Cosmet. Chem., 1949, 1, 311-326.

[8] Ruckenstein, E. Microemulsions, Macroemulsions, and the Bancroft rule. Langmuir 1996, 12, 6351-6353.

[9] Binks, B.P. Modern Aspects of Emulsion Science, Royal Society of Chemistry, Cambridge, UK, 1998. 
[10] Verdinelli, V.; Vuano, B.; Schultz, P.C.; Messina, P.V. Inversion Properties of n-Alkane Phosphonic Acids Stabilized Emulsions: HLB Dependence. J. Disper. Sci. and Technol., 2009, 30, 1538-1544 .

[11] Salager, J.L.; Minana-Perez, M.; Perez-Sanchez, M.; RamirezGouveia, M.; Rojas, C.I. Surfactant-oil-water systems near the affinity inversion. 3. The 2 kinds of emulsion inversion. J. Disper. Sci. Technol., 1983, 4, 313-329.

[12] Vaessen, G.E.J.; Stein, H.N. Predicting catastrophic phase inversion on the basis of droplet coalescence kinetics. Langmuir 1996, $12,875-882$.

[13] Vaessen, G.E.J.; Stein, H.N. The applicability of catastrophe theory to emulsion phase inversion. J. Colloid Interface Sci., 1995, 176, 378-387.

[14] Smith, H.; Johnson, G.K. Temperature-dependence of emulsion morphologies and the dispersion morphology diagram. 2. 3-phase emulsions. J. Phys. Chem., 1995, 99, 10853-10857 .

[15] Ruckenstein, E. Microemulsions, Macroemulsions, and the Bancroft rule. Langmuir 1996, 12, 6351-6353.

[16] Binks, B.P. Relationship between Microemulsion Phase Behavior and Macroemulsion Type in Systems Containing Nonionic Surfactant. Langmuir 1993, 9, 25-28 .

[17] Ravera, F.; Ferrari, M.; Ligieri, L. Adsorption and partitioning of surfactants in liquid-liquid systems. Adv. Colloid Interface Sci., 2000, 88, 129-177.

[18] Wikipedia. Triton X-100. http://en.wikipedia.org/wiki/Triton X-100 (accessed January 21, 2008).
[19] Göbel, J.G.; Joppien, G.R.; Dynamic interfacial tensions of aqueous triton X-100 solutions in contact with air, cyclohexane, n-heptane, and n-hexadecane J. Colloid Interface Sci., 1997, 191, 30-37.

[20] Campanelli, J.R.; Wang, X. Dynamic interfacial tension of surfactant mixtures at liquid-liquid interfaces $J$. Colloid Interface Sci., 1999, 213, 340-351.

[21] Dow Surfactants, Triton X-100. http://msdssearch.dow.com/ PublishedLiteratureDOWCOM/dh 05b7/0901b803805b7f48.pdf? filepath $=$ surfactants/pdfs/noreg/119-01882.pdf\&fromPage $=$ GetDoc (accessed July 13, 2011).

[22] ISO 304, Surface active agents - Determination of surface tension by drawing up liquid films.1978.

[23] Provencher, S.W. Inverse problems in polymer characterization Direct analysis of polydispersity with photon correlation spectroscopy. Makromol. Chem., 1979, 180, 201-209.

[24] Atkins, P.; de Paula, J. Physical Chemistry, 8th Ed.; Oxford University Press, 2006, p. 145

[25] Cheung, H.M.; Qutubuddin, S.; Edwards, R.V.; Mann Jr., J.A. Light-scattering study of oil-in-water microemulsions - Corrections for interactions. Langmuir 1987, 3, 744-752.

[26] Walstra, P. Principles of emulsion formation. Chem. Eng. Sci., 1993, 48, 333-349.

[27] McBain, J.W. Mobility of Highly-charged Micelles. Trans. Faraday Soc., 1913, 9, 99-101.

(C) Laven et al.; Licensee Bentham Open.

This is an open access article licensed under the terms of the Creative Commons Attribution Non-Commercial License (http://creativecommons.org/licenses/by-nc/3.0/) which permits unrestricted, non-commercial use, distribution and reproduction in any medium, provided the work is properly cited. 